\title{
A local defect resonance for linear and nonlinear ultrasonic thermography
}

\author{
by I. Solodov, M. Rahammer and G. Busse
}

Department of nondestructive testing, Institute of Polymer Technology, University of Stuttgart, 70569 Stuttgart, Germany, igor.solodov@ikt.uni-stuttgart.de

\begin{abstract}
An efficient wave-defect interaction is the key to a high thermal response of flaws in ultrasonic thermography. To selectively enhance defect vibrations a concept of local defect resonance is developed and applied to ultrasonic activation of defects. The frequency match between the defect resonance frequency and the probing ultrasonic wave results in a substantial rise of a local defect temperature. The defect resonance is accompanied by depletion of the excitation frequency vibration due to nonlinear frequency conversion to higher harmonics. The local generation of higher frequency components provides a high thermal defect response in such an acoustically nonlinear thermography mode.
\end{abstract}

\section{Introduction}

Elastic wave-defect interaction is a background of ultrasonic nondestructive evaluation (NDE) of materials and industrial components. In conventional (linear) ultrasonic NDE, it is responsible for sound attenuation and scattering that results in the wave amplitude and phase variations as indicators of the presence of defects. The efficiency of the interaction becomes particularly crucial for ultrasonic NDE-techniques, which require an elevated acoustic power such as nonlinear ultrasonic spectroscopy [1] and ultrasonic thermography [2]. In the latter case, to provide high-amplitude vibrations special horn-type transducers aimed for ultrasonic welding are usually combined with kW-power supply.

Further increase in vibration amplitude is attained by choosing the excitation frequency equal to the natural frequency of the specimen $[3,4]$. However, for reliable visualisation of the defect care should taken to assure that the defect is outside the nodal areas of the standing wave pattern. To diminish the effect of nodal lines and to prevent "missing" the defect, it was suggested to excite the specimens at multiple natural frequencies instead of using a singlefrequency excitation [5]. Particular frequency selection was attributed to avoiding the areas of low-sensitivity due to nodal line patterns [5] or to providing particular displacement pattern (clapping or rubbing) of a vibrating crack [6].

A step to optimize the defect thermal response by using continuous frequency variation was made in [7] where the frequency sweep mode of ultrasound thermography was proposed. In this mode, the frequency is swept over a certain range (few $\mathrm{kHz}$ ) and a local temperature as a function of frequency is monitored for each pixel of the thermography image. The frequency with a maximum thermal response of the defect is then selected and used for ultrasonic excitation. The sweep mode was used as a standalone method and was also combined with lock-in and burst thermography measurements [8].

An enhancement in crack detectability by producing a wide-band excitation ("acoustic chaos") was reported in $[9,10]$. Chaotic vibrations were produced in ultrasonic source due to contact nonlinearity caused by "clapping" of the interface between the horn transducer and the specimen. For the same acoustic power injected in a cracked Alspecimen, the crack thermal response for chaotic excitation was 2-3 times higher than that for a monochromatic ultrasonic source. The authors acknowledged that the origin of the effect "is unclear" [10] and might be related to elimination of vibrational nodes of the sample or correlated with the presence of higher frequencies [9].

The alternative approaches, used in acoustics of gas bubbles in liquids [11] introduce a resonance frequency of the bubble as a key factor to increase an ultrasonic response of the insonified inclusion. The discovery of anomalous nonlinear ultrasonic response of medical contrast agents whose local nonlinearity enhanced remarkably due to resonance vibrations of incorporated gel bubbles made a breakthrough in ultrasonic medical diagnostics [12].

Likewise, the opportunity of a resonance interaction of ultrasound with defects in solids was theoretically analysed in [13] for plate waves. The model of a resonance inclusion in soil was also applied to development of the nonlinear seismo-acoustic land mine detection methodology [14]. The phenomenology of "resonant" defects was used by us to describe the nonlinear dynamics of flaws in solid materials [15]. Recent numerical simulations and shearographic imaging demonstrated a modal vibration structure for delaminations [16] and flat-bottom holes [17] in composites.

In this paper, a consistent way to enhance acoustic and thermal defect responses is suggested by using selective ultrasonic activation of defects based on the concept of local defect resonance (LDR) [18]. The LDR provides an efficient energy pumping from the wave directly to the defect, which results in a high local vibration amplitude and enhancement of thermal defect response readily measurable even for a sub-Watt acoustic input. As the excitation amplitude increases, the LDR-"amplifier" exhibits transition to nonlinear regime with higher harmonic generation solely in the defects area. The local generation of higher frequency components provides a further enhancement of defect thermal response in such an acoustically nonlinear thermography mode. 


\section{LDR phenomenology and defect resonance frequencies}

The LDR concept is based on the fact that inclusion of a defect leads to a local drop of stiffness for a certain mass of the material in this area, which should manifest in a particular characteristic frequency $\left(f_{0}\right)$ of the defect. To illustrate the reduction in local stiffness we first use a model of the defect as a spherical cavity of radius $R$. For a stress normal to the cavity surface, the local stiffness $\left(E_{L}\right)$ of such a defect is given by [19]:

$$
E_{L}=\frac{2(1-2 \sigma)}{3(1-\sigma)} E_{I} \approx 0.3 E_{I}
$$

According to (1), the stiffness of intact material $\left(E_{I}\right)$ reduces about three times for materials with Poisson`s ratio $\sigma \approx 0.3$. Similar estimations for a disk-like crack of elliptical cross-section with semiaxes $R$ (radius of the disk) and $d$ (half of maximal opening) show that the local Young's modulus is [19]: $E_{L} \approx(d / R) E_{I}$. Assuming that for realistic cracked defects $R \sim\left(10^{-3}-10^{-2}\right) \mathrm{m}$ and $d \sim 10^{-6} \mathrm{~m}$, the local drop in stiffness can be very substantial: $E_{L} / E_{I} \sim\left(10^{-3}-10^{-4}\right)$.

By deriving the local rigidity of the void and vibrating mass of the material, from (1) the estimate of the LDR frequency for a spherical cavity in a solid takes the form similar to the one in a liquid:

$$
f_{0} \approx \sqrt{\frac{E_{I}}{16 \pi^{3} \rho R^{2}}} .
$$

By using conventional parameters for a composite material ( $E_{I} \approx 30 \mathrm{GPa} ; \rho \approx 2 \cdot 10^{3} \mathrm{~kg} / \mathrm{m}^{3}$ ) for a void of 1 mm-radius we obtain: $f_{0} \approx 174 \mathrm{kHz}$.

For such a typical defect in composites as a sub-surface delamination, the local resonance is apparently attributed to the resonance vibrations of the material layer (thickness $D$ ) above the defect and identified with a flexural resonance of a plate of thickness $D$ clamped around the boundary. By using a circular plate approximation (radius $a$ ) the estimate of the fundamental resonance frequency of the delamination is given by [18]:

$$
f_{0} \cong \frac{3.2 D}{2 a^{2}} \sqrt{\frac{E}{12 \rho\left(1-\sigma^{2}\right)}} .
$$

For a $2 \mathrm{~cm}$-delamination at $1 \mathrm{~mm}$ depth in a typical composite material with $(E \approx 30 \mathrm{GPa} ; \sigma \approx 0.3$ and $\rho \approx 2000 \mathrm{~kg} / \mathrm{m}^{3}$ ), from (3) the fundamental LDR is expected at $f_{0} \approx 19 \mathrm{kHz}$. Both evaluations given above show that the LDR frequencies belong to ultrasonic frequency range and are located quite distantly from the specimen natural frequencies (sub- and low-kHz range) that facilitates their experimental observation.

\section{Experimental observation of LDR}

A direct way to experimentally reveal a local defect resonance is to measure an individual contribution of each point of the specimen in its overall frequency response in a wide frequency range. For this purpose, an ultrasonic excitation by a wide-band piezoelectric transducer is combined with a laser vibrometer C-scanning of the specimen surface. It enables to probe and indicate all possible resonances in every point of the specimen.

An example of application of such an approach is shown in Fig. 1 for an in-plane oval delamination $\left(25 \times 18 \mathrm{~mm}^{2}\right)$ in a glass fibre-reinforced composite (GFRP) plate $\left(200 \times 25 \times 2.5 \mathrm{~mm}^{3}\right)$. A piezoelectric transducer embedded in the plate was used for a wide-band $(400 \mathrm{~Hz}-40 \mathrm{kHz})$ excitation of flexural waves. For this purpose, a pseudo-random input electrical voltage (flat frequency response within $0-50 \mathrm{kHz}$ (see inset in Fig. 1); amplitude $1-70 \mathrm{~V}$ ) was applied to the transducer. The out-of-plane particle velocity components and the wave vibration pattern in the specimen were measured and visualized by a scanning laser vibrometer (Polytec 3001S).

The origin of each maximum in Fig. 1 was verified by imaging the wave pattern in the specimen at the corresponding frequency. The pattern in Fig. 2 (a) illustrates one of the specimen length resonances, which are located in the frequency range below $10 \mathrm{kHz}$ in Fig. 1. Similarly, a series of peaks in the frequency band 10-18 kHz was found to be associated with the specimen width resonances. The specimen vibration pattern measured at $\sim 20 \mathrm{kHz}-$ resonance 
(Fig. 2 (b)) reveals a strong enhancement (more than $20 \mathrm{~dB}$ ) of the vibration amplitude locally in the defect area and is identified as a fundamental defect resonance. The zoom-in 2D-image (Fig. 3 (a)) shows that the resonance response is inhomogeneous over the defect area with a particularly high-amplitude in the left (core) part of the delamination. Multiple higher-order resonances with characteristic nodal lines in the defect area were also found in the frequency range up to $40 \mathrm{kHz}$ (Fig. 3 (b)).

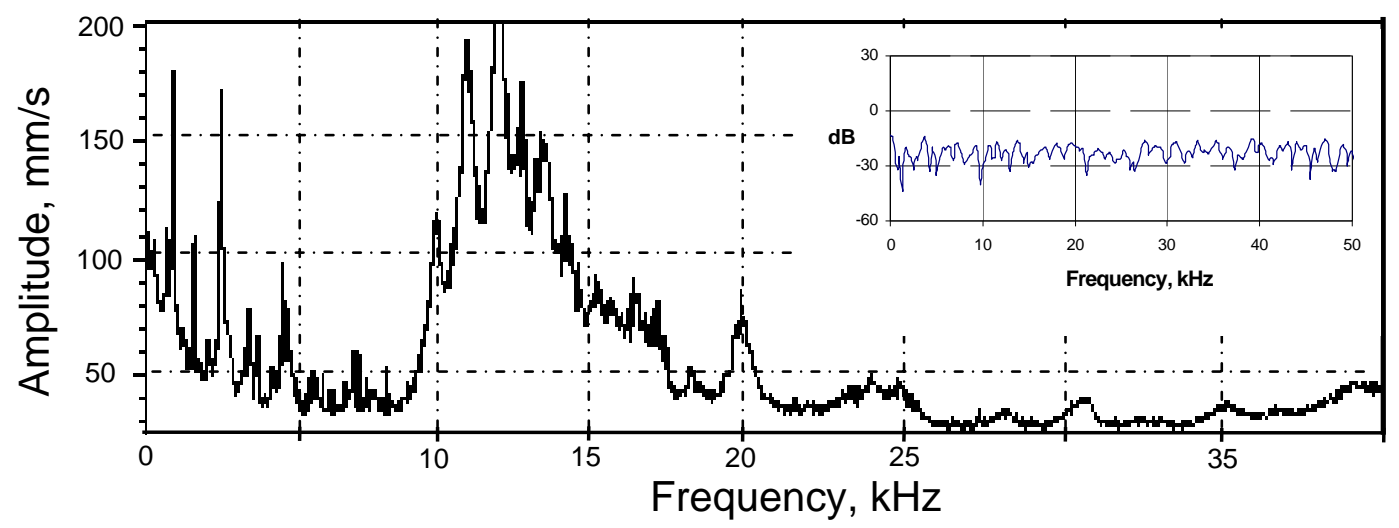

Fig. 1. Frequency response of GFRP specimen with inter-ply delamination measured with a scanning laser vibrometer.
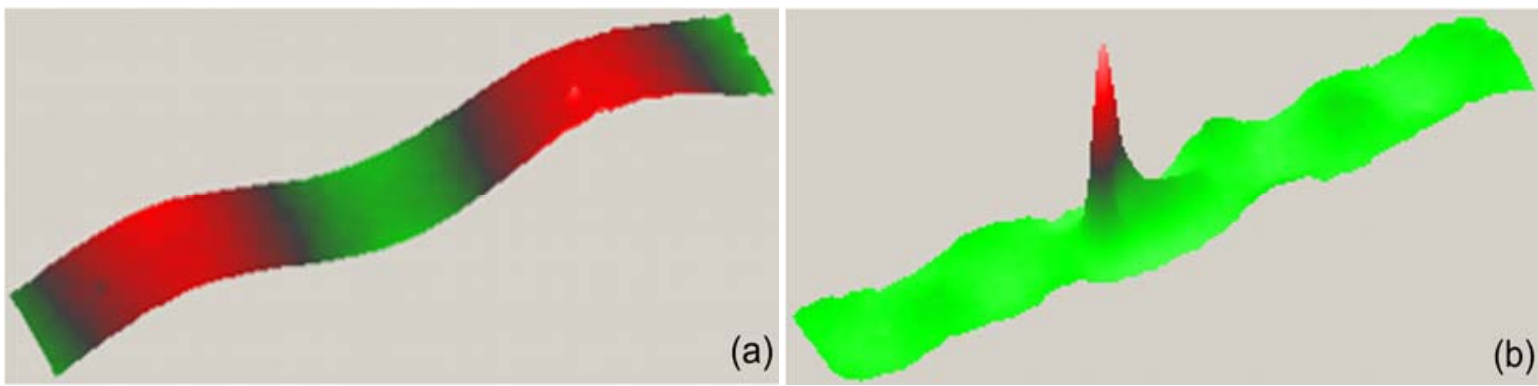

Fig. 2 (a), (b). Laser vibrometry of vibration pattern for GFRP-strip with a local delamination: (a)-driving at eigen-frequency $6.8 \mathrm{kHz}$; (b)-frequency $19.75 \mathrm{kHz}$.
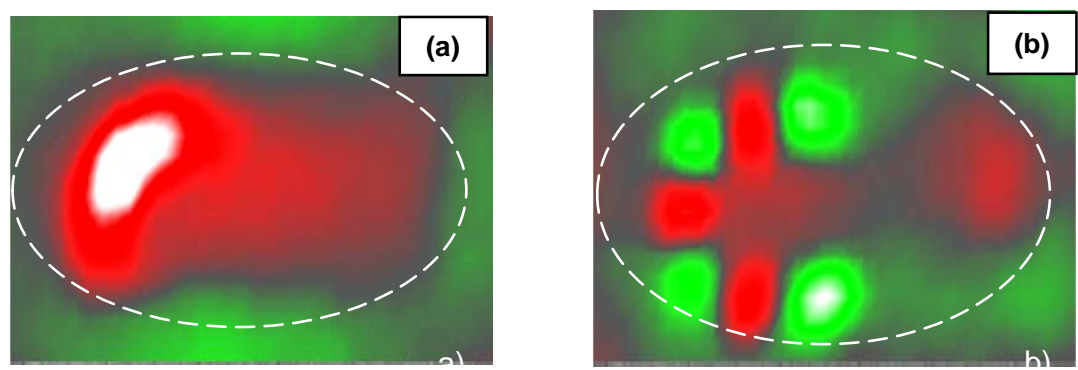

Fig. 3 (a), (b). Zoom-in 2D-images of the vibration modes for the fundamental (a) and higher-order (b) local defect resonances.

For elastic constants of the material derived from the measurements of longitudinal and shear wave velocities: $E \approx 30 \mathrm{GPa} ; \sigma \approx 0.3$ and $\rho \approx 2500 \mathrm{~kg} / \mathrm{m}^{3}$; for the delamination parameters $D \approx 1 \mathrm{~mm} ; a \approx 9 \mathrm{~mm}$, from (3) we obtain $f_{0} \approx 20.7 \mathrm{kHz}$, that is in a good agreement with the experimental value of $\approx 20 \pm 1 \mathrm{kHz}$.

In further experiments, wide-band (vacuum attached) piezo-transducers (ISI SYS GmbH) were applied for ultrasonic excitation of the specimens. The electronic unit comprised an HP 33120A function generator (bandwidth up to $15 \mathrm{MHz}$ ) and a high voltage amplifier HVA 3/450 (SI GmbH). Another example of LDR-methodology is shown in Fig. 4 
(left) for imaging of a $50 \mu \mathrm{m}$-wide crack in a GFR-concrete plate $\left(450 \times 100 \times 10 \mathrm{~mm}^{3}\right)$. The LDR-frequency is substantially lower in this case $(4.188 \mathrm{kHz}$ for $\mathrm{a} \sim 10 \mathrm{~cm}$ long crack) while more than $20 \mathrm{~dB}$ dynamic range of the image proves a feasibility of the LDR- approach. Higher LDR frequencies are expected in high-stiffness materials, such as carbon fibre-reinforced composite (CFRP) ( $E>100 \mathrm{GPa}$ ). This is illustrated in Fig. 4 (right): to observe the LDR image of an impact area in a CFRP specimen the driving frequency had to be raised up to $66.22 \mathrm{kHz}$.
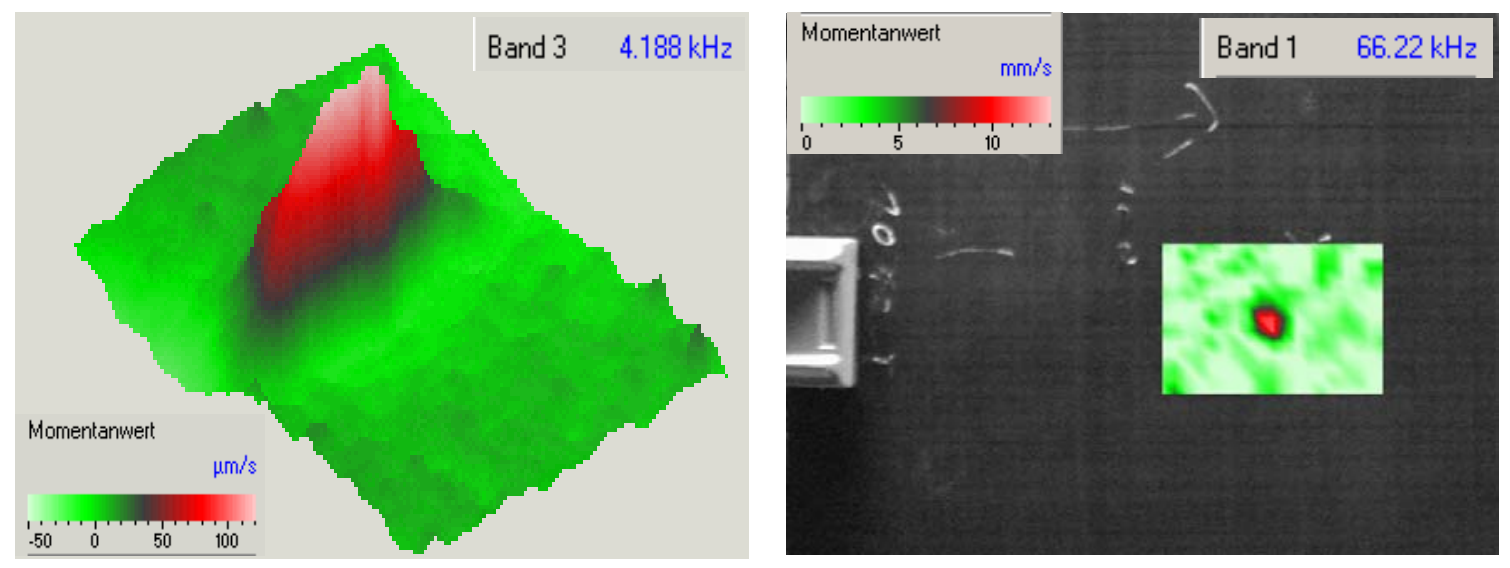

Fig. 4. Left: LDR imaging of a crack in a GFR-concrete plate (size of the imaged area is $150 \times 100 \mathrm{~mm}^{2}$ ); right: $L D R$ in a circular impact area (radius $\sim 15 \mathrm{~mm}$ ) in a CFRP plate $\left(250 \times 200 \times 1 \mathrm{~mm}^{3}\right)$.

\section{Ultrasonic LDR thermography}

\subsection{Ultrasonic thermography via linear LDR}

In ultrasonic thermography, the defect thermal response is caused by a local dissipation of mechanical energy, which is converted into heat. In an acoustically linear regime, the variation of mechanical energy $(\Delta W)$ can be estimated by using phenomenology of internal friction forces. For monochromatic ultrasonic vibrations $\varepsilon=\varepsilon_{0} \exp (j \omega t)$ in such "inelastic" materials, stress-strain relation acquires an imaginary term: $\sigma=E \Sigma+j \omega E$ “ $\varepsilon$, which causes a phase shift between $\sigma$ and $\varepsilon$ that makes the relation hysteretic. The power developed by internal friction forces, which is converted into heat in a unit volume of material, is: $\Delta W / \Delta t \sim E{ }^{\prime 2} \omega^{2} \varepsilon_{0}^{2}$, so that ultrasonic heating is proportional to the square of both vibration amplitude and frequency.

Therefore, the use of LDR, which strongly intensifies local defect vibrations (increase in $\varepsilon_{0}$ ), is beneficial for enhancing the efficiency and sensitivity of ultrasonic thermography. These benefits are illustrated in Figs. 5, 6, where the thermal responses of the delamination are visualized (Fig. 5) and measured (Fig. 6) for the same input amplitudes (input voltage $50 \mathrm{~V}$ ) but different frequencies.
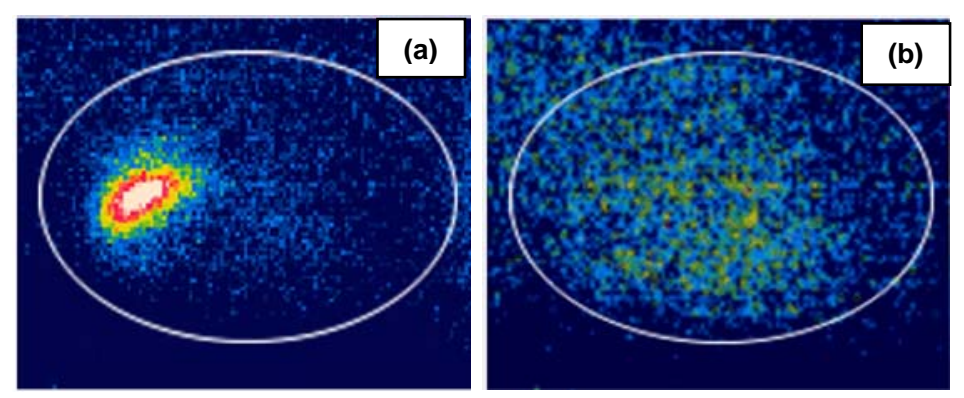

Fig. 5 (a), (b). Thermographic images of the delamination in GFRP at fundamental defect resonance frequency $(\sim 20 \mathrm{kHz},(\mathrm{a}))$ and at a frequency of one of the specimen length resonance $(6.8 \mathrm{kHz},(\mathrm{b}))$. 


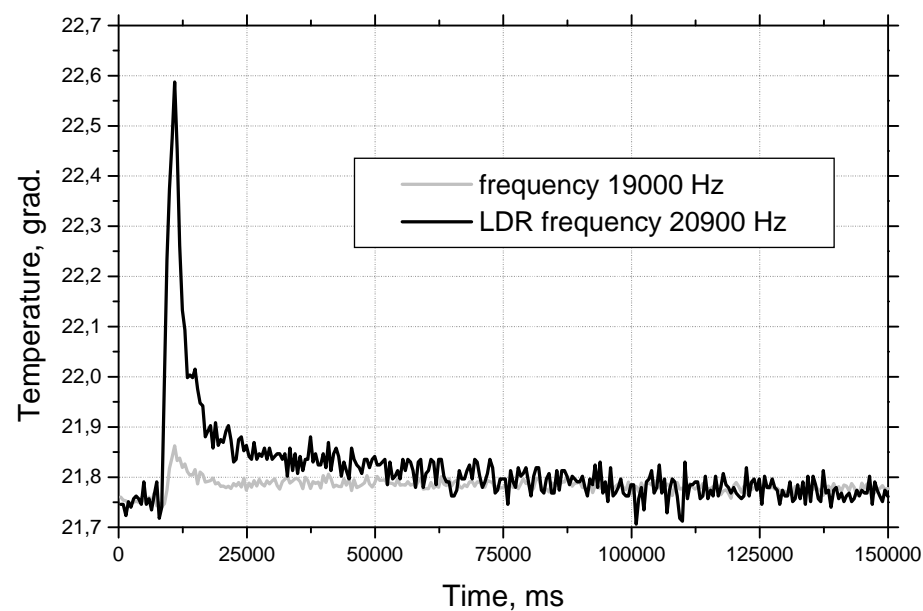

Fig. 6. Thermal responses of delamination at LDRfrequency $(20900 \mathrm{~Hz})$ and outside the LDR bandwidth
The thermographic image of the defect taken in the vicinity of the resonance frequency $20 \mathrm{kHz}$ (Fig. 5 (a)) demonstrates that the heating is mainly produced in the core part of the delamination where maximum vibration amplitude is observed (Fig. 3 (a)). This illustrates a defectselective nature of the LDR-thermography. The temperature variation in this area (for insonification time $3 \mathrm{~s}$ ) (Fig. 6) confirms the resonance character of the effect: At the LDR central frequency (20900 $\mathrm{Hz}$, Fig. 5 (a)), the temperature rise is almost an order of magnitude higher than that outside the LDR bandwidth. For larger deviation of the driving frequency from LDR (including the specimen natural frequencies), the thermal response of the defect was barely measurable with the IR-camera (IRCAM Equus $327 \mathrm{~K}$, sensitivity $\approx 20 \mathrm{mK}$ ).

\subsection{Nonlinear LDR ultrasonic thermography}

At low amplitude of monochromatic excitation, LDR manifests as a conventional (linear) resonance and enhances the defect vibrations at the resonance frequency. As was shown above, a local stiffness in the area of a small crack can reduce dramatically: $E_{L} \approx(d / R) E_{I}$, so that for the stress applied, the crack acts as a local strain "amplifier" by $(R / d)$ factor. As a result, even at moderate ultrasonic excitation level, the faces of the cracked defect start "clapping" and its vibrations become strongly nonlinear [20]. Subject to both nonlinearity and LDR, the defect exhibits the properties of an acoustically nonlinear LDR.

A strong increase in defect nonlinearity due to LDR is illustrated in Fig. 7 for another delamination in a GFRP specimen. The zoom-in frequency response of a delamination obtained for a wide-band excitation (Fig. 7 (a)) reveals the LDR-frequency $f_{0} \approx 5050 \mathrm{~Hz}$. For driving frequency outside the LDR-bandwidth ( $f \approx 4840 \mathrm{~Hz}$ ), the defect vibration spectrum comprises mainly the excitation frequency component and some higher harmonics, whose amplitude is rather small even at input voltage raised up to $50 \mathrm{~V}$ (Fig. 7 (b)). As the driving frequency matches the LDR-frequency, a drastic enhancement of the higher harmonic $(\mathrm{HH})$ spectrum is observed (Fig. 7 (c)). The HH-measurements within LDRbandwidth (e.g. Fig. 8) show that the resonance induced increase in the $\mathrm{HH}$-amplitudes can be as high as $30-40 \mathrm{~dB}$.

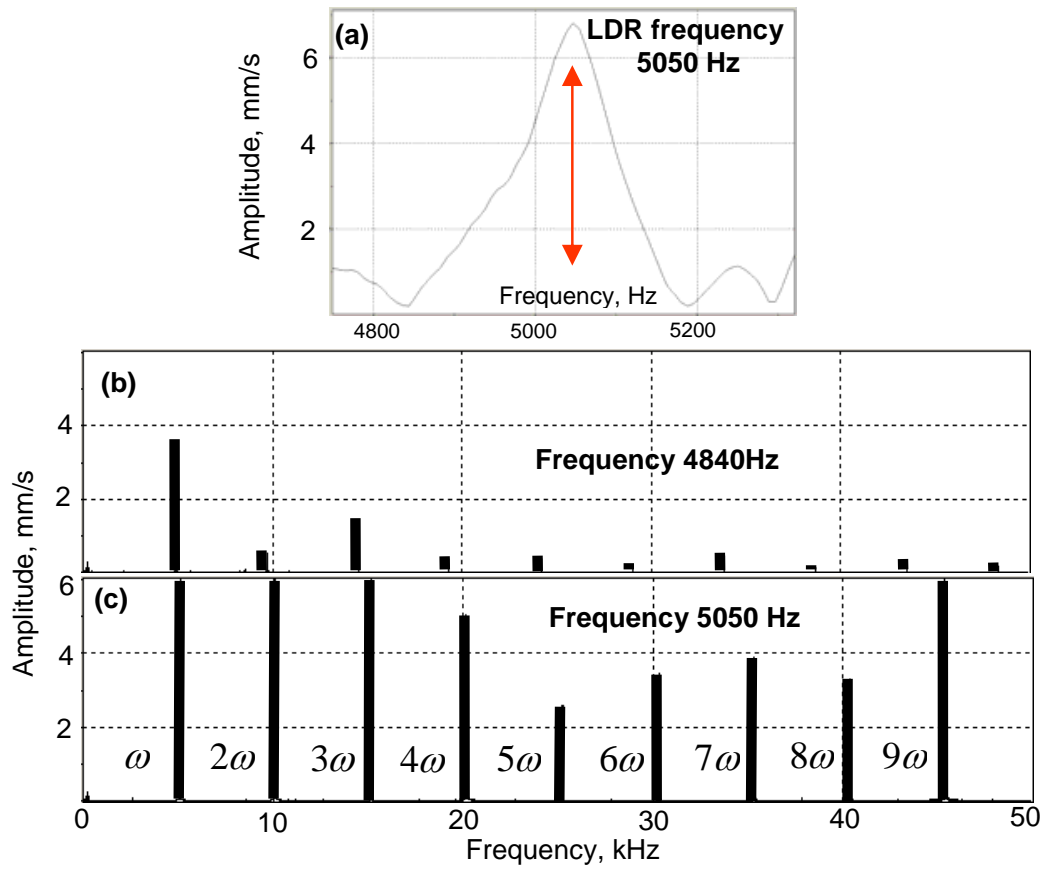

Fig. 7 (a), (b), (c). Zoom-in frequency response of LDR for a delamination in GFRP (a); HH-spectrum outside (b) and at LDR-frequency (c). 


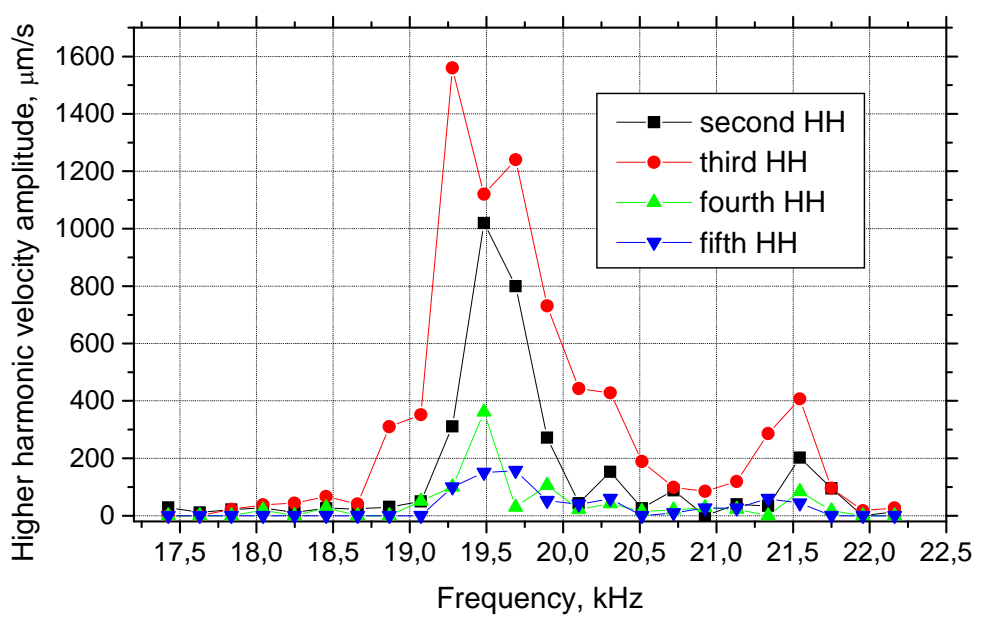

Fig. 8. Resonance increase of the higher harmonic amplitudes in the frequency band of LDR for a crack in a carbon fibrereinforced rod.
As was shown above, for a linear elastic defect response, ultrasoundinduced heating is proportional to the square of excitation frequency. However, the frequency increase of injected ultrasound is accompanied by the higher ultrasonic attenuation in the material and enhancement of the background heating. The nonlinear LDR suggests a unique opportunity to generate the higher frequency components directly in the defect and thus to enhance a local heating of the defect selectively. In the nonlinear LDR mode, the defect generates multiple spectral components (see [21] and Fig. 7), which extend the local excitation spectrum to a much higher frequency range. Each spectral line of the high frequency vibrations contributes to the defect thermal response, which is enhanced due to both

the high number of the nonlinear spectral components and their higher frequencies.

A feasibility of such a nonlinear thermography mode is demonstrated in Figs. 9-10.

The laser vibrometry measurements of vibration velocity averaged over the thermally active area of the delamination in the GFRP plate (Fig. 5, a) show that as the defect excitation level increases (input voltage $>60 \mathrm{~V}$ ), the vibrations at the fundamental excitation frequency (squared vibration velocity proportional to a "linear" elastic energy (Fig. 9, left) saturate and then reduce considerably. This might be caused by the amplitude-induced frequency shift of LDR and the generation of nonlinear spectral components in the delamination area (nonlinear distortion). The relevance of the latter factor is seen in Fig. 9, right: the sum of squared $\mathrm{HH}$-vibration velocities ("nonlinear" elastic energy) measured in the frequency band up to $1 \mathrm{MHz}$ (over $47 \mathrm{HH}$ ) increases strongly at the higher excitation level. According to Fig. 9, about half of the "loss" of elastic energy at the excitation frequency is converted in the higher harmonic vibrations, which take place in the defect area.

The "depletion" of the vibrations at fundamental frequency, however, does not affect the thermal response of the defect (Fig. 10, left), which increases steadily over the whole range of excitation level (up to $90 \mathrm{~V}$, Fig. 10, left). An additional source of heating comes from nonlinear vibrations of the defect: the ultrasound-induced temperature (Fig. 10, left) rises synchronously with the growth of nonlinear elastic energy (Fig. 9, right). The nonlinearity of the defect, therefore, contributes substantially to its thermal response and enhances the sensitivity of ultrasonic thermography.

This effect is clearly illustrated in Fig. 10, right, where the delamination thermal response is plotted as a function of the amplitude of fundamental excitation frequency component. In a low-amplitude range (acoustically linear LDR), ultrasonic heating grows quadratically, that is in a full accord with conclusions of section 4.1. Transition into nonlinear LDR is accompanied by depletion of the fundamental frequency vibrations; decrease in its amplitude beyond the inflection point in Fig. 9, left results in the "turn" of the curve in Fig. 10. Despite the fundamental frequency amplitude decreases above the turning point, the defect thermal response continues to grow. The "internal source" for further heating is produced by the higher frequency components generated within the defects due its nonlinearity.
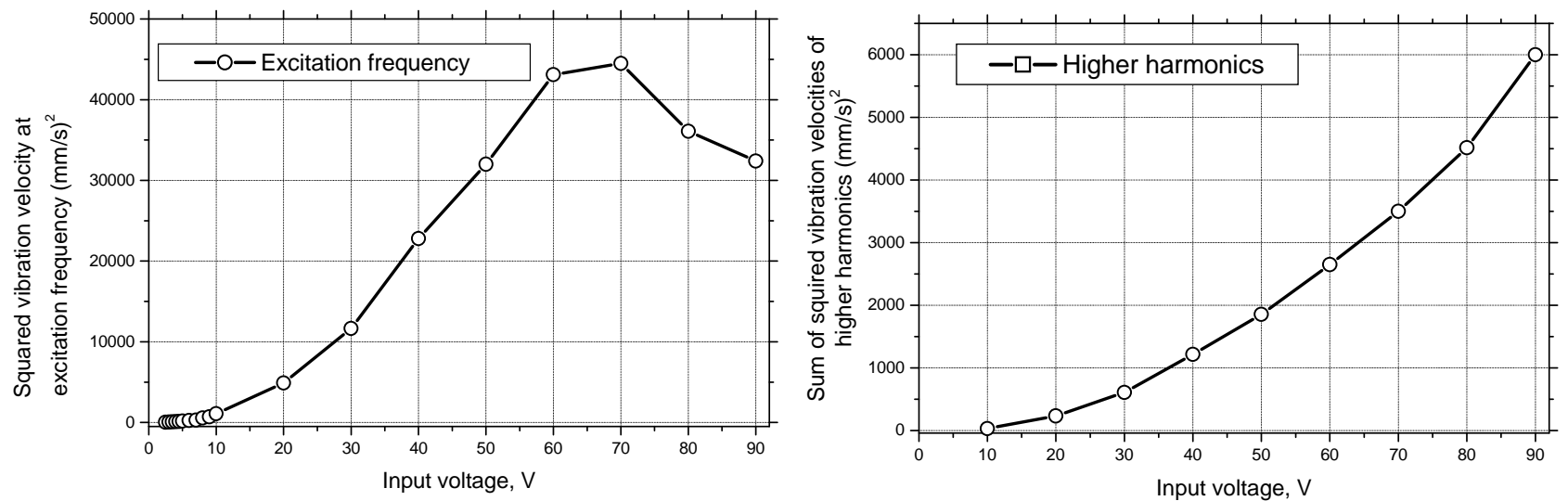

Fig. 9. Laser vibrometry measurements of vibrations at fundamental excitation frequency (LDR $20900 \mathrm{~Hz}$ ) (left) and higher harmonics (right) as functions of excitation level. 
To quantify the efficiency of ultrasound-heat conversion under the LDR conditions, the input acoustic power was evaluated by measuring particle vibration velocity in the excitation area with laser vibrometer. It was found that at $90 \mathrm{~V}$ voltage applied to conventional piezo-transducer attached to GFRP-specimen, the acoustic power generated is $\sim 0.2 \mathrm{~W}$. According to Fig. 10, this results in temperature rise of $0.7 \mathrm{~K}$ in the delamination area for $2 \mathrm{sec}$ ultrasonic exposure. In other LDR experiments with delaminations in GFRP, the temperature elevations of $\sim(1-3) \mathrm{K}$ were measured for input acoustic power in the range of $\sim(0.3-0.5) \mathrm{W}$.
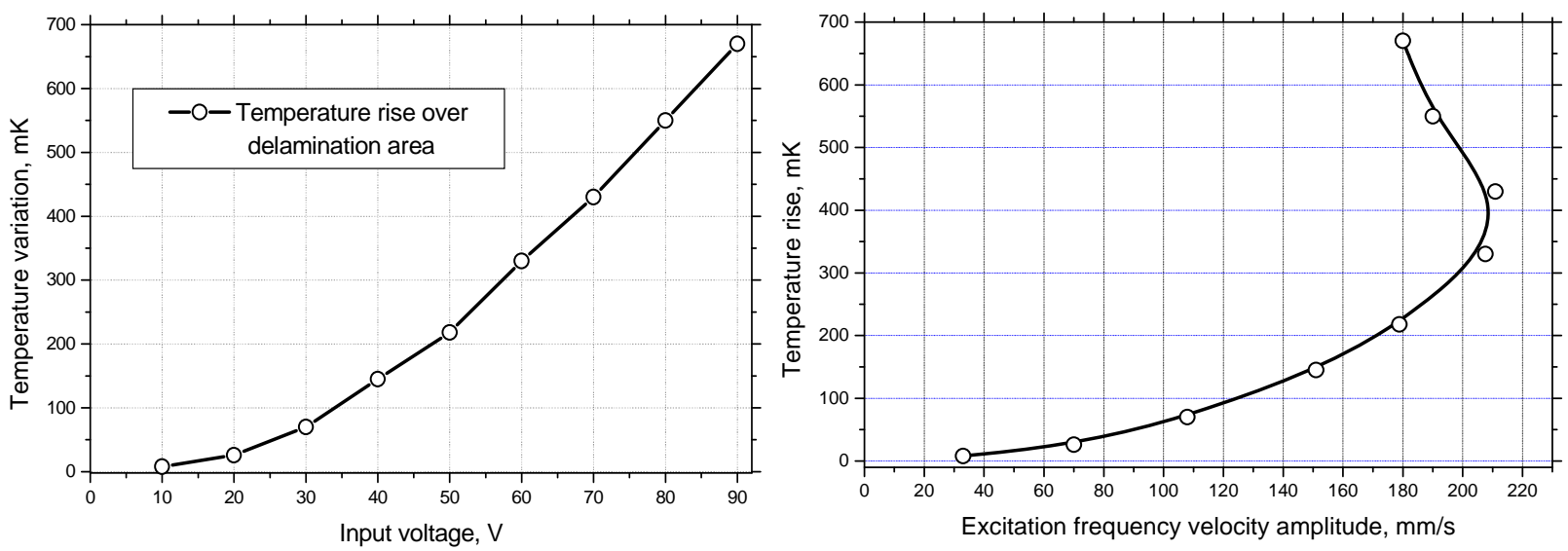

Fig. 10: Thermal response of the delamination area in GFRP as a function of excitation level (left) and fundamental excitation frequency velocity amplitude (right). Ultrasonic exposure time is 2 sec.

\section{Conclusions}

It has been experimentally demonstrated that the concept of local mechanical resonance is applicable to defects in solid materials. For medium size flaws in composites, the resonance frequencies of defects lie in $\mathrm{kHz}$ frequency range.To optimize acoustic wave-defect interaction, a frequency match between the local defect resonance (LDR) response and the probing ultrasonic wave is required. In this condition, the acoustic energy is delivered directly to the defect (local vibration amplitude increases by 20-40 dB) that requires substantially lower ultrasonic power to activate the defect in ultrasonic thermography. For input acoustic power below $1 \mathrm{~W}$, the temperature rise about $1 \mathrm{~K}$ is normally measured for LDR of delaminations in composite materials. The LDR also diminishes heating of the intact part of the specimen, enhances the sensitivity, contrast and selectivity of defect thermographic imaging. Even at moderate level of ultrasonic excitation at LDR-frequency, the cracked defects exhibit the properties of an acoustically nonlinear LDR. Transition into nonlinear LDR is accompanied by decrease in the amplitude of the fundamental frequency vibrations that, however, does not affect the thermal response of the defect, which increases steadily. The "internal source" for heating is produced by the higher frequency components generated within the defects due its nonlinearity, which therefore provides a substantial increase of defect thermal response in such an acoustically nonlinear mode of LDR-thermography.

\section{REFERENCES}

[1] Van Abeele K.E-A., Sutin A., Carmeliet J., Johnson P. "Micro-damage diagnostics using nonlinear elastic wave spectroscopy (NEWS)". NDT\&E International, vol. 34, pp. 239-248, 2001.

[2] Mignogna R.B., Green Jr. R.E., Duke J.C., Henneke II E.G., and Reifsnider K.L. "Thermographic investigation of high-power ultrasonic heating in materials". Ultrasonics, vol. 19, N 4, pp. 159-163, 1981.

[3] Fillinger L., Zaitsev V., Gusev V., and Castagnede B. "Wave self-modulation in an acoustic resonator due to selfinduced transparency". Europhys. Lett., vol. 76, N2, pp. 229-235, 2006.

[4] Renshaw J., Holland S., and Thompson R.B. "Measurement of crack opening stresses and crack closure stress profiles from heat generation in vibrating cracks". Appl Phys Lett., vol. 93, N8, 081914, 2008.

[5] Homma C., Rothenfusser M., Baumann J., and Shannon R. "Study of the heat generation mechanism in acoustic thermography". AIP Conf. Proc., vol. 820, pp. 566-573, 2006.

[6] Rothenfuser M. and Homma C. "Acoustic thermography: Vibrational modes of cracks and the mechanism of heat generation". AIP Conf. Proc., vol. 760, pp. 624-631, 2005.

[7] Gleiter, A., Spießberger C., Zweschper T., Busse G., "Improved ultrasound activated lockin-thermography using frequency analysis of material defects". QIRT Journal, vol. 4, N2, pp. 155-164, 2007.

[8] Spießberger C., Gleiter A., Busse G. "Ultrasound Sweep Thermography (UST) as a new method of nondestructive testing". In: Emerging Technologies in Non-Destructive Testing, eds. G. Busse et. al. Taylor\&Francis Group, pp. 199-203, 2008. 
[9] Han X., Loggins V., Zhi Zheng, Favro L.D., Thomas R.L. "Mechanical model for the generation of acoustic chaos in sonic infrared imaging". Appl. Phys. Lett., vol. 85, N 8, pp. 1332-1334, 2004.

[10] Han X., Zhi Zheng, Wei Li, Md. Sawar Islam, Janping Lu, Loggins V., Yitamben E., Favro L.D., Newaz G., Thomas R.L. "Acoustic chaos for enhanced detectability of cracks by sonic infrared imaging". J. Appl. Phys., vol. 95, N 7, pp. 3792-3797, 2004.

[11] Lauterborn W. "Numerical investigation of nonlinear oscillations of gas bubbles in liquids". J. Acoust. Soc. Am. Vol. 59, N2, pp. 283-293, 1976.

[12] Frinking P.J.A., Bouakaz A., Kirkhorn J., Ten Cate F.J., and de Jong N. "Ultrasound contrast imaging: current and new potential methods". Ultrasound Med. Biol., vol. 26, pp. 965-975, 2000.

[13] Rokhlin S.I. "Resonance phenomena of Lamb wave scattering by a finite crack in a solid layer". J. Acoust. Soc. Am., vol. 69, pp. 922-928, 1981.

[14] Zagrai A., Donskoy D., Chudnovsky A., and Golovin E. "Micro- and macroscale damage detection using the nonlinear acoustic vibro-modulation technique". Research in Nondestructive Evaluation, vol. 19, pp. 104-128, 2008.

[15] Solodov I., Wackerl J., Pfleiderer K., and Busse G. "Nonlinear self-modulation and subharmonic acoustic spectroscopy for damage detection and location". Appl. Phys. Lett., vol. 84, N26, pp. 5386-5388, 2004.

[16] Sarens B., Verstraeten B., Glorieux Ch., Kalogiannakis G., and Van Hemelrijck D. "Investigation of contact acoustic nonlinearity in delamination by shearographic imaging and finite difference modeling". IEEE Trans. Ultrason, Ferroel., Freq. Control, vol. 57, N6, pp. 1383-1395, 2010.

[17] De Angelis G., Meo M., Almond D.P., Pickering S.G., Angioni S.L. "A new technique to detect defect size and depth in composite structures using digital shearography and unconstrained optimization". NDT\&E International, vol. 45, pp. 91-96, 2012.

[18] Solodov I., Yuxing Bai, Bekgulyan S., Busse G. "A local defect resonance to enhance acoustic wave-defect interaction in ultrasonic nondestructive testing". Appl. Phys. Lett., vol. 99, 211911, 2011.

[19] Mavko G., Mukerji T., Dvorkin J. "The rock physics handbook". Cambridge University Press, 2003.

[20] Solodov I., Krohn N., Busse G. "CAN: An example of nonclassical nonlinearity in solids". Ultrasonics, vol. 40, pp. 621-625, 2002.

[21] Solodov I., Pfleiderer K., and Busse G. "Nonlinear Acoustic NDE: Inherent potential of complete nonclassical spectra". In: Universality of Nonclassical Nonlinearity with Application to NDE and Ultrasonics, Ed. P. Delsanto, Chapter 29, pp. 465-484, Springer Verlag, New York, USA, 2006. 